\title{
Resistance of Mycobacterium tuberculosis to antibiotics in Lao PDR: first multicentric study conducted in 3 hospitals
}

\author{
Vibol lem ${ }^{1 \dagger}$, Silaphet Somphavong ${ }^{1,2 \dagger}$, Yves Buisson ${ }^{2}$, Nicolas Steenkeste ${ }^{1}$, Franck Breysse ${ }^{3}$, Monique Chomarat $^{3}$, \\ Phannasinh Sylavanh ${ }^{4}$, Phouratsamy Nanthavong ${ }^{5}$, Alain Rajoharison ${ }^{6}$, Jean-Luc Berland ${ }^{6 *}$ \\ and Phimpha Paboriboune ${ }^{1}$
}

\begin{abstract}
Background: It is estimated that Lao People's Democratic Republic (Lao PDR) ranks fifth among the seven countries most affected by TB in the WHO Western Pacific Region. However, because of late implementation of mycobacterial culture, no study on resistance to anti-TB drugs had been performed yet. The objective of this study was to document drug resistance rate among patients hospitalized for pulmonary TB in threeprovinces of Lao PDR

Methods: A cross-sectional study was conducted in three sites, one central and two regional hospitals, from April to November 2010. For each TB suspected patient sputum smear microscopy and culture on Lowenstein-Jensen media were performed. GenoType ${ }^{\oplus}$ MTBDRplus assay was used to test the susceptibility to isoniazid (INH) and rifampicin (RMP), GenoType ${ }^{\circledast}$ MTBDRsl for second-line drugs and GenoType ${ }^{\circledR}$ Mycobacterium CMAS for nontuberculous mycobacteria (NTM).

Results: Out of 104 positive culture on Lowenstein-Jensen, 87 (83.6\%) were M. tuberculosis and 17 (16.4\%) were NTM. Of 73 new TB cases, 5 isolates (6.8\%) were resistant to INH. Of 14 previously treated cases, 2 isolates (14.3\%) were resistant to INH and one isolate was XDR.
\end{abstract}

Conclusion: Despite an overall rate of resistance still moderate, the frequency of mutations conferring $\mathrm{INH}$ monoresistance and identification of the first strain of XDR require strengthening surveillance of drug resistant tuberculosis in Lao PDR.

Keywords: Multidrug resistant TB, Non Tuberculosis Mycobacteria, Multicentric

\section{Background}

The control of tuberculosis (TB) in developing countries is hampered by the progression of drug-resistant TB, especially multi-drug resistance (MDR). MDR-TB strains are resistant to at least the first-line drugs isoniazid (INH) and rifampicin (RMP), which significantly reduces the therapeutic arsenal. The weighted rates of resistance in the world population are estimated among new cases at $17 \%$ for any resistance and $2.9 \%$ for MDR, and among previously treated cases at $35 \%$ for any resistance and $15.3 \%$ for

\footnotetext{
* Correspondence: jean-luc.berland@fondation-merieux.org

${ }^{\dagger}$ Equal contributors

${ }^{6}$ Laboratoire des Pathogènes Émergents, Fondation Mérieux, 21 Avenue Garnier, 69007, Lyon, France

Full list of author information is available at the end of the article
}

MDR [1]. The World Health Organization (WHO) estimates that each year 440,000 new cases of MDR-TB are emerging globally, and 150,000 patients with MDR-TB die. More worrying is the emergence of extensively drugresistant TB (XDR-TB) caused by MDR strains that also resist to fluoroquinolones and second-line anti-TB injectable drugs (amikacin, kanamycin or capreomycin). WHO estimates that 25,000 cases of XDR-TB emerge each year worldwide. Unfortunately, only a small fraction of patients infected by MDR or XDR-TB can access a screening by culture, drug-susceptibility testing and appropriate management [2]. The use of new rapid molecular tests for first-line drug resistance can dramatically reduce the delays to detect MDR-TB and adapt the treatment. The good performances of molecular line probe assays have led 
WHO to approve their use for rapid screening of patient at risk of MDR-TB [3].

In Lao PDR, the burden of TB remains high: it is the seventh leading cause of death in adults. In 2009, the prevalence of all forms of TB was estimated at 289/100 000 , the incidence at $151 / 100000$ and the mortality at 24/ 100000 [4]. The DOTS was implemented in 1995 by the National Tuberculosis Control Program (NTCP) [5]. The recommended treatment uses combinations of 5 drugs at fixed doses (S: streptomycin, R: rifampicin, $\mathrm{H}$ : isoniazid, $\mathrm{Z}$ : pyrazinamide, E: Ethambutol) corresponding to the following protocols: 2RHZE/6HE for new TB cases, 2RHZE/ $4 \mathrm{RH}$ for TB-HIV co-infections and 2RHZES/6RHZE for re-treatment. The therapeutic success rate of new smear positive cases exceeds 90\% since 2005 and reached 93\% in 2009. However, a retrospective cohort study of TB patients showed that the $85 \%$ survival rate was lower than the expected $91 \%$ from official statistics and that followup should be strengthened to improve patient compliance and allow contact tracing and detection of new cases $[4,6]$. The implementation of the first mycobacterial cultures in 2009 has made feasible the anti-TB drug susceptibility testing [7]. The creation of a national reference laboratory for TB allowed the realization of a first national prevalence survey in 2010-2011. But more efforts should be implemented to achieve the Millennium Development Goal 6 of halving TB prevalence and reverse its incidence by 2015 [8].

In this context, this study aimed to provide a preliminary assessment of the rate of $M$. tuberculosis resistance to isoniazid (INH), rifampicin (RMP) and second-line drugs in Lao PDR using a molecular line probe assay.

\section{Methods}

\section{Design of the study}

We conducted a cross-sectional study from April to November 2010 in a central hospital in Vientiane (Mahosot hospital) and two provincial hospitals (Thakhek and Luang Prabang). Patients enrolled in this study were directly admitted to one of the 3 hospitals and not transferred from another health care facility.

\section{Study population}

All patients over 15 years attending the pneumology units with symptoms suggestive of TB were included, regardless of their HIV status, as well as patients given a TB treatment. These patients were considered (i) as new cases if never previously treated or beginning the treatment within the last month prior inclusion, (ii) as previously treated cases if the treatment started more than one month before the inclusion [2]. Inclusion criteria were cough for over two weeks, or bloody sputum, or suggestive radiological signs, irrespective to HIV infection. Chest radiography or tuberculin skin tests were not systematically performed.

\section{Specimen processing}

Two sputum samples were collected for each patient, the first during the consultation, the second the next morning upon awakening. Screening for acid-fast bacilli (AFB) was locally performed in each hospital on ZiehlNeelsen stained smears. Sputum were then stored at $4{ }^{\circ} \mathrm{C}$ and sent to Rodolphe Mérieux Laboratory in Vientiane once a week for culture and drug susceptibility testing.

Specimens were digested and decontaminated by the $\mathrm{N}$-acetyl-L-cystein-sodium hydroxide (NALC-NaOH) procedure [9], then inoculated on two slants of LowensteinJensen medium incubated at $37^{\circ} \mathrm{C}$. Cultures were examined once a week for 80 days. Any suspect colony confirmed as AFB by microscopy was identified and tested for antibiotic resistance.

\section{Identification of M. tuberculosis and drug susceptibility testing}

When several cultures were positive for the same patient, only one was tested to detect drug resistances. The GenoType ${ }^{\bullet}$ MTBDRplus (Hain lifescience Gmbh, Germany) was used for $M$. tuberculosis complex identification and INH and/or RMP resistance detection according to manufacturer's instructions.

In case of resistance to INH and/or RMP, the Genotype ${ }^{\odot}$ MTBDRsl (Hain lifescience Gmbh, Germany) was then performed to detect the resistances to second-line antibiotics.

Isolates not belonging to the $M$. tuberculosis complex were identified using the Genotype ${ }^{ø}$ Mycobacterium CM/ AS (Hain lifescience Gmbh, Germany) that can differentiate 13 species of clinically relevant non-tuberculous mycobacteria (NTM).

\section{Statistical analysis}

Data were entered with EPI data 3.1 and analyzed with Stata 8.0. The chi2 test was used to compare qualitative variables with a significance level of 0.05 .

\section{Ethical clearance}

This study was approved by the National Ethics Committee of Lao PDR and the National Tuberculosis Control Program Center (\# 201/NECHR).

A written informed consent for participation in the study was obtained from participants. Only patients over 15 years old with signed informed consent were included in the study.

\section{Results}

\section{Mycobacterial culture and identification}

Eighty-seven isolates (83.6\%) were identified as belonging to the $M$. tuberculosis complex, evenly distributed between the three sites $(\mathrm{p}=0.1)$. The 17 remaining strains (16.4\%) were NTM of which 10 were identified as 
M. fortuitum (8), M. scrofulaceum (1) and M. abscessus (1). Out of the $87 M$. tuberculosis strains, 74 were isolated from new cases while 13 were isolated from previously treated cases (Table 1).

\section{Characteristics of patients}

Among the 87 patients with at least one positive culture for M. tuberculosis, 34 (39.1\%) lived in Vientiane, 31 (35.6\%) in Thakhek and 22 (25.3\%) in Luang Prabang. Sex ratio (M/F) was 1.3 and mean age 48 years. Most of the patients were new cases (85\%). The 13(15\%) patients previously treated or treated for more than 1 month were unevenly distributed among the three sites: 63\% Vientiane, 19\% in Luang Prabang and 19\% in Thakhek. HIV status was only known for 5 patients; none of whom were HIV positive (Table 2).

\section{Drug resistance}

\section{Resistance to first line drugs}

Among the $87 M$. tuberculosis isolates, 8 (9.2\%) were found resistant: 7 mono-resistant to INH and 1 to both INH and RMP; 3 (13.6\%) in Luang Prabang, 3 (9.7\%) in Thakhek and 2 (5.9\%) in Vientiane, without significant difference between the three sites $(\mathrm{p}=0.5)$. Five of them were isolated from new cases and were resistant to $\mathrm{INH}$ : two mutated in the katG gene (S315T), two in the inhA gene $(\mathrm{C} 15 \mathrm{~T})$ and one in both genes katG (S315T) and inhA (C15T) (Table 3). The three remaining isolates were from previously treated cases: they were resistant to INH by S315T mutation in $k a t G$ or by deletion of this gene. For this last strain (165 LP), none of the $4 \mathrm{katG}$ locus probes on the strip reacted; further sequencing showed neither katG gene nor katG promoter could be amplified using several inner and outer primers, reflecting the complete deletion of this gene. To further analyze this strain, $r p o B$, pncA, inh $A$ and $a h p C$ genes were sequenced. No mutation was observed in rpoB, pncA and inhA genes, suggesting RMP and pyrazinamide susceptibility. The $a h p C$ gene sequencing revealed the ambiguous base calls in the $-51,-52$ and -57 promoter positions. Therefore, amplicons were cloned and 20 of them were sequenced. Interestingly, the results showed the coexistence of three different mutational profiles, none with more than one mutated site. This strain was isolated from an 82-old patient who had received a TB treatment six years ago and was considered cured. He relapsed in 2012 and died quickly, before the culture results are positive. The third strain (175 TK) was also resistant to RMP by mutation in the rpoB gene (H526Y) and was therefore MDR (Table 3).

\section{Drug resistance}

Resistance to second-line drugs

Out of the five new cases displaying resistance mutations, two INH-resistant strains were also resistant to aminoglycosides and cyclic peptides showing a C1402T mutation on the rrs gene. Out of the three previously treated cases, two strains were resistant to at least one second-line drug (Table 3).

The MDR-TB isolate (175-TK) displayed three further mutations: $\mathrm{M} 306 \mathrm{~V}$ in $\mathrm{embB}$ gene, $\mathrm{A} 90 \mathrm{~V}$ in gyrA gene and $\mathrm{C} 1402 \mathrm{~T}$ in rrs gene. This strain was sent to Korean Institute of Tuberculosis (KIT, Seoul, Korea) to perform a conventional antibiogram with the second line drugs. It turned out that this strain was indeed resistant to RMP, INH, ethambutol, fluoroquinolones, streptomycin and aminoglycosides/cyclic peptides, and it was therefore an XDR strain. The patient (175 TK) was a 31 years old woman, diagnosed pulmonary TB in 2004 and treated with a first line regimen (2RHZE/6HE) for 8 months. She was considered clinically cured with smear microscopy negative at fifth month of treatment. She relapsed in 2007 and received a second-line treatment (2RHZES/6RHZE) for 8 months followed by INH monotherapy for 3 years. In 2010, the diagnosis of chronic pulmonary TB resistant to treatment was bacteriologically confirmed. She then received a combination therapy of HZE, kanamycin and ofloxacin for 6 months (Table 4).

\section{Discussion}

The recent implementation of mycobacterial culture in Laos PDR had made possible the necessary monitoring of resistance to anti-TB drugs. This multicenter study aimed to provide preliminary data on the rate of resistance to first-line anti-TB, especially INH and RMP. Patient enrolled were outpatients, not referred from peripheral clinics, thus not being at higher risk of drug resistant. The results show an overall rate of resistance of approximately $10 \%$, homogeneously distributed among the 3 sites. It reached $6.8 \%$ among new cases and $21.4 \%$ among previously treated cases. Despite a sampling not as robust as national survey, this study shows similar

Table 1 Results of culture and identification

\begin{tabular}{|c|c|c|c|c|c|}
\hline Number of patients with & Total & Vientiane & Thakhek & Luang Prabang & $\mathbf{p}$ \\
\hline$\geq 1$ positive culture (\%) & 104 & $39(37.5)$ & $38(36.5)$ & $27(26)$ & \\
\hline M. tuberculosis complex & 87 & $34(39.1)$ & $31(35.6)$ & $22(25.3)$ & 0.7 \\
\hline NTM $^{*}$ & 17 & $5(29.4)$ & $7(41.2)$ & $5(29.4)$ & \\
\hline
\end{tabular}

*NTM non-tuberculous mycobacteria ( 8 M. fortuitum, 1 M. scrofulaceum, 1 M. abscessus and 7 unidentified). 
Table 2 Characteristics of patients

\begin{tabular}{|c|c|c|c|c|c|}
\hline & $\begin{array}{c}\text { Total } \\
87\end{array}$ & $\begin{array}{l}\text { Mahosot } \\
34(39 \%)\end{array}$ & $\begin{array}{l}\text { Thakhek } \\
31(36 \%)\end{array}$ & $\begin{array}{l}\text { Luang Prabang } \\
22(25 \%)\end{array}$ & $\mathbf{p}$ \\
\hline \multicolumn{6}{|l|}{ Gender } \\
\hline Male & 50 & $21(42)$ & $17(34)$ & $12(24)$ & \multirow[t]{3}{*}{0.8} \\
\hline Female & 37 & $13(35.2)$ & $14(37.8)$ & $10(27)$ & \\
\hline Average age (IC95\%) & $48(45-52)$ & $52(45-59)$ & $46(41-51)$ & $46(38-55)$ & \\
\hline \multicolumn{6}{|l|}{ Age groups } \\
\hline$\cdot \geq 15-25<$ years & 7 & $3(42.9)$ & $1(14.2)$ & $3(42.9)$ & \multirow{3}{*}{0.06} \\
\hline$\cdot \geq 25-55 \leq$ years & 48 & $14(29.2)$ & $23(47.9)$ & $11(22.9)$ & \\
\hline$\cdot>55$ years & 32 & $17(53.2)$ & $7(21.9)$ & $8(25)$ & \\
\hline \multicolumn{6}{|l|}{ TB history } \\
\hline Never treated or $<1$ months & 74 & $27(36.5)$ & $28(37.8)$ & $19(25.7)$ & \multirow[t]{2}{*}{$<0}$. \\
\hline Previously treated or $\geq 1$ months & 13 & $7(53.8)$ & $3(23.1)$ & $3(23.1$ & \\
\hline \multicolumn{6}{|l|}{ HIV status } \\
\hline Positive & 0 & - & - & - & \multirow{3}{*}{0.1} \\
\hline Negative & 5 & $1(20)$ & $4(80)$ & $0(0)$ & \\
\hline Unknown & 82 & $33(40.3)$ & $27(32.9)$ & $22(26.8)$ & \\
\hline
\end{tabular}

rates compared to neighboring countries: Cambodia, 10\% and $16.6 \%$ in the 2001-2002 national survey [10], and Myanmar, 10\% and 30.2\% in the 2002 national survey [11]. However, it remains well below the rates reported in Thailand, 20.9\% and 53.6\% in 1996 [12], in Vietnam, 26.3\% and $62.9 \%$ in 2001 [13] and in China, 27.9\% and 60.3\% in a meta-analysis covering the period 1993-2011 [14].

All the resistant isolates of this study showed mutations conferring resistance to INH. The only isolate resistant to RMP, also carrying mutations conferring resistance to second-line antibiotics, came out to be an XDR. Even though no strictly MDR strain was isolated during the study, these results show the existence of resistance mutations in $\mathrm{TB}$ isolates that may lead to compromise the effectiveness of treatment regimens recommended by the NTCP.

This study presents a number of limitations. The patient sampling was performed in three large hospitals which do not represent the whole country. A recruitment in remote district hospitals where patient followup and compliance to DOTS are less easy to perform [6] might have revealed higher levels of resistance. The HIV status of patients was unknown for $92 \%$ of patients, indicating that the recommendations for routine screening of HIV-TB co-infection had not been yet systematically applied. The exclusive use of a molecular test for rapid

Table 3 Mutation patterns of eight isolates of $M$. tuberculosis

\begin{tabular}{|c|c|c|c|c|c|c|}
\hline \multirow{2}{*}{$\begin{array}{l}\text { Patient } \\
\text { number } \\
\text { (hospital) }\end{array}$} & \multirow{2}{*}{$\begin{array}{l}\text { Previously } \\
\text { treated } \\
\text { case }\end{array}$} & \multicolumn{5}{|c|}{ Mutations conferring resistance to } \\
\hline & & INH & RMP & FLQ & $A G / C P$ & EMB \\
\hline \multirow[b]{2}{*}{$425(L P)$} & \multirow[b]{2}{*}{ No } & katG S315T & \multirow[b]{2}{*}{ - } & \multirow[b]{2}{*}{-} & \multirow[b]{2}{*}{ - } & \multirow[t]{2}{*}{-} \\
\hline & & inhA C15T & & & & \\
\hline $472(\mathrm{TK})$ & No & $\operatorname{inh} A$ C15T & - & - & - & - \\
\hline 977 (MV) & No & katG S315T & - & - & - & - \\
\hline $454(\mathrm{MV})$ & Yes & katG S315T & - & - & - & - \\
\hline $446(L P)$ & No & inhA C15T & - & - & rrs C1402T & - \\
\hline $480(\mathrm{TK})$ & No & katG S315T & - & - & rrs C1402T & - \\
\hline $165(\mathrm{LP})$ & Yes & katG deleted & - & - & rrs C1402T & - \\
\hline $175(\mathrm{TK})$ & Yes & katG S315T & rpoB H526Y & gyrA A90V & rrs C1402T & embB M306V \\
\hline \multicolumn{2}{|c|}{ Total } & 8 & 1 & 1 & 4 & 1 \\
\hline
\end{tabular}

LP Luang Prabang, MV Mahosot Vientiane, TK Thakhek, INH isionazid, RMP rifampycin, FLQ fluoroquinolones, $A G / C P$ aminoglycosides, cyclic peptides, EMB ethambutol.

(-) Presence of all wild type probes and none mutation probe observed. 
Table 4 History of the three previously treated patients

\begin{tabular}{|c|c|c|c|}
\hline & \multicolumn{3}{|c|}{ Patient number (hospital) } \\
\hline & 454 (MV) & 165 (LP) & 175 (TK) \\
\hline Gender / age (years) & $M / 54$ & $M / 82$ & $F / 31$ \\
\hline date of $1^{\text {st }}$ diagnosis of TB & 2003 & 2004 & 2004 \\
\hline $1^{\text {st }}$ anti-TB regimen & 2RHZE/6HE & 2RHZE/6HE & 2RHZE/6HE \\
\hline Outcome of $1^{\text {st }}$ TB treatment & recovery & recovery & failure \\
\hline Date of $\mathrm{TB}$ relapse & 2010 & 2012 & 2007 \\
\hline Date of initiation of treatment & 2012 & a & 2007 \\
\hline Current anti-TB regimen & 2RHZES & a & 2RHZES/6RHZE \\
\hline Duration of treatment & 2 months & a & 8 months $+\mathrm{H} 3$ years \\
\hline Compliance with TB treatment & unknown & a & high \\
\hline Clinical response to treatment & poor & a & poor (b) \\
\hline
\end{tabular}

a: the patient died before the culture results and did not receive the second-line treatment.

b: chronic pulmonary tuberculosis resistant to treatment.

species identification of mycobacterial isolates and for detection of antibiotic resistance may also lead to underestimate INH resistance. This line probe assay is part of rapid methods recommended by WHO for detection of MDR strains as a substitute for conventional methods which require 4 to 6 weeks. It can even be performed directly from sputum samples found positive at microscopy. Rapid detection of resistance to RMP is a major issue because it can be considered a surrogate marker for MDR strains [15]. According to a meta-analysis of 14 comparative studies for RMP and 15 for INH, performances of the GenoType MTBDR assay are considered satisfactory, except for the detection of INH resistance whose sensitivity approaches $90 \%$ with the MTBDRplus assay [16]. Some strains phenotypically resistant to INH are found genetically susceptible, possibly because of mutations involving genes other than inhA and katG [17]. Similarly, the MTBDRsl assay showed an excellent specificity in detecting resistance to second-line drugs, but a variable sensitivity, especially for the detection of resistance to fluoroquinolones and ethambutol $[18,19]$. The inclusion of new alleles is recommended in order to reduce the proportion of false negative results [20].

The eight resistant strains isolated in our study all showed a mutation conferring resistance to INH: 5 in $k a t G, 2$ in $\operatorname{inh} A$ and the remaining 1 in both $k a t G$ and inhA. Interestingly, the three inhA promoter mutants were observed in new cases,. A study conducted during a 9-years period in San Francisco showed that strains with a katG S315T or inhA promoter mutation were more likely to spread than otherwise mutated strains [21]. However, mutations in the inhA promoter are more common among INH-monoresistant isolates than among MDR isolates [22]. It is in all cases a C15T mutation which is associated with a low level of resistance [23]. This mutation confers co-resistance to INH and ethionamide [24].
Of the 6 strains mutated in $k a t G, 5$ had the S315T mutation, currently considered as a risk factor for further development of MDR-TB [25]. The last one had a complete deletion of this gene, a mechanism rarely found associated with clinical INH-resistance [26,27]. By sequencing, the distinction of three clones each carrying one peculiar mutation in the $\operatorname{ahpC}$ gene is consistent with the hypothesis that up regulation mutations in the $a h p C$ promoter compensate for the loss of catalaseperoxidase activity encoded by the katG gene [28].

The only case of RMP-resistance was due to a mutation H526Y in the rpoB gene. With codons 531 and 516, this point mutation is one of the most frequently associated with resistance to RMP [29].

Four strains, two from new cases and two from previously treated cases, exhibited a C1402T mutation in the rrs gene, presumed to confer resistance to aminoglycosides and cyclic peptides: this mutation was found associated with high levels of resistance to capreomycin, but with low levels of resistance to kanamycin and amikacin [30]. Regarding the two new cases, this mutation strongly suggests a primary resistance whose origin is unclear, the only aminoglycoside antibiotic widely used in Lao PDR as firstline treatment until the 2000s being the streptomycin.

Despite the progress made by the NTCP to achieve the WHO objectives, implementation of the DOTS strategy in Lao PDR is limited by the remoteness of the target population to health centers, the difficult follow-up of patients under TB treatment and the lack of compliance. The recent development of mycobacterial culture and the use of line probe assays have allowed identifying the species of the $M$. tuberculosis complex and, for the first time, to detect resistances to anti-TB drugs. This preliminary investigation shows that resistance mutations, especially against $\mathrm{INH}$, are present among the new cases, some of which may evolve towards MDR. 
The isolation of an XDR strain from a patient enrolled in a provincial hospital is the result of chance. This strain acquired resistance as a result of successive treatments; XDR being the consequence of adding only 2 drugs never received to a previous treatment failure, instead of 5 as recommended by WHO. In Cambodia, of 101 MDR strains isolated between 2007 and 2009, only one was found XDR [31]. However, this must sound like a threat because the XDR strains have the ability to spread in the population by intra-community transmission [32] and justifies the strengthening and extension of the laboratory network for tuberculosis in the different provinces of Lao PDR. The use of line probe assays such as MTBDR tests should now be recommended to rapidly detect and treat without delay the MDR-TB cases.

\section{Conclusion}

The frequency of primary resistance to INH and identification of the first XDR strain strongly justify to strengthen and expand the screening of resistant TB in the provinces of Lao PDR.

\section{Competing interests}

The authors declare that they have no competing interests.

\section{Authors' contributions}

$\mathrm{VI}, \mathrm{SS}, J \mathrm{JB}, \mathrm{NS}, \mathrm{FB}, \mathrm{MC}, \mathrm{PS}, \mathrm{PN}, \mathrm{AR}, \mathrm{YB}, \mathrm{PP}$ contributed to experimental procedures.

$\mathrm{VI}, \mathrm{SS}, \mathrm{JLB}, \mathrm{NS}, \mathrm{MC}, \mathrm{FB}, \mathrm{PS}, \mathrm{YB}, \mathrm{PP}$ drafted and critically revised the manuscript. All authors read and approved manuscript.

\section{Acknowledgements}

The authors thank the National Tuberculosis Control Program Center for supporting the project, the staff of pneumology departments (Mahosot, Thakhek, Luang Prabang) for sample collection and shipping, and the technicians of the Centre Infectiologie Christophe Mérieux du Laos for their active participation.

The work was funded by a grant from Fondation Mérieux (Lyon, France) and Institut de la Francophonie pour la Médecine Tropicale (IFMT, Vientiane, Lao PDR).

\section{Author details}

${ }^{1}$ Ministry of health, Centre d'Infectiologie Christophe Mérieux du Laos, Samsenthai Road, Kaoyot Village, Sisathanak District, Vientiane Capital, Lao PDR. ${ }^{2}$ Institut de la Francophonie pour la Médecine Tropicale, Ban Kaognoth, rue Samsènethai, Vientiane Capital, Lao PDR. ${ }^{3}$ Laboratoire de Bactériologie, Groupement Hospitalier Sud, Lyon, France. ${ }^{4}$ The National Tuberculosis Control Program Center, Vientiane, Lao PDR. ${ }^{5}$ Service de Pneumologie, Hopital Mahosot, Vientiane Capital, Lao PDR. '́ Laboratoire des Pathogènes Émergents, Fondation Mérieux, 21 Avenue Garnier, 69007, Lyon, France.

Received: 16 October 2012 Accepted: 11 June 2013

Published: 19 June 2013

\section{References}

1. WHO/IUATLD, WHO/IUATLD Global Project on Anti-tuberculosis Drug Resistance Surveillance: Anti-tuberculosis drug resistance in the world, Fourth global report. Geneva; 2008

2. WHO/HTM/TB: Multidrug and extensively drug-resistant TB (M/XDR-TB), 2010 global report on surveillance and response. Geneva; 2010.

3. Nathanson E, Nunn P, Uplekar M, et al: MDR tuberculosis-critical steps for prevention and control. N Engl J Med 2010, 363:1050-1058.

4. WHO: Global Tuberculosis Control Report. Geneva; 2009.

5. Arnadottir TH, Soukaseum H, Vangvichit P, Bounmala S, Vos E: Prevalence and annual risk of tuberculosis infection in Laos. Int I Tuberc Lung Dis 2001, 5:391-399.
6. Barennes $H$, Keophithoun $T$, Nguyen $T H$, Strobel M, Odermatt P: Survival and health status of DOTS tuberculosis patients in rural Lao PDR. BMC Infect Dis 2010, 10:265.

7. Thammavong C, Paboriboune P, Bouchard B, et al: Bleach treatment of sputum samples aids pulmonary tuberculosis screening among HIVinfected patients in Laos. Int I Tuberc Lung Dis 2011, 15:1353-1358.

8. Nair N, Wares F, Sahub S: Tuberculosis in the WHO South-East Asia Region. Bull World Health Organ 2010, 88:164-165.

9. Kubica GP, Dye WE, Cohn ML, Middlebrook G: Sputum digestion and decontamination with $\mathrm{N}$-acetyl-L-cysteine-sodium hydroxyde for culture of mycobacteria. Am Rev Respir Dis 1963, 87:775-779.

10. Yamada N, Saorith K, Yamakami K, et al: The national tuberculosis drug resistance survey in Cambodia, 2000-2001. Int J Tuberc Lung Dis 2007, 11:1321-1327.

11. Ti T, Lwin T, Mar TT, et al: National anti-tuberculosis drug resistance survey, 2002, in Myanmar. Int J Tuberc Lung Dis 2006, 10:1111-1116.

12. Riantawan P, Punnotok J, Chaisuksuwan R, Pransujarit V: Resistance of Mycobacterium tuberculosis to antituberculosis drugs in the Central Region of Thailand, 1996. Int J Tuberc Lung Dis 1998, 2:616-620.

13. Huong NT, Lan NT, Cobelens FG, et al: Antituberculosis drug resistance in the south of Vietnam: prevalence and trends. J Infect Dis 2006 194:1226-1232.

14. Yang Y, Li X, Zhou F, Jin Q, Gao L: Prevalence of drug-resistant tuberculosis in Mainland China: systematic review and meta-analysis. PLoS One 2011, 6:e20343.

15. Ling DI, Zwerling AA, Pai M: GenoType MTBDR assays for the diagnosis of multidrug-resistant tuberculosis: a meta-analysis. Eur Respir J 2008 32:1165-1174

16. Tolani MP, D'souza DT, Mistry NF: Drug resistance mutations and heteroresistance detected using the GenoType MTBDRplus assay and their implication for treatment outcomes in patients from Mumbai, India. BMC Infect Dis 2012, 12:9.

17. Brossier F, Veziris N, Aubry A, Jarlier V, Sougakoff W: Detection by GenoType MTBDRsl test of complex mechanisms of resistance to second-line drugs and ethambutol in multidrug-resistant Mycobacterium tuberculosis complex isolates. J Clin Microbiol 2010, 48:1683-1689.

18. Kiet VS, Lan NT, An DD, et al: Evaluation of the MTBDRsl test for detection of second-line-drug resistance in Mycobacterium tuberculosis. J Clin Microbiol 2010, 48:2934-2939.

19. Huang WL, Chi TL, Wu MH, Jou R: Performance assessment of the GenoType MTBDRsl test and DNA sequencing for detection of secondline and ethambutol drug resistance among patients infected with multidrug-resistant Mycobacterium tuberculosis. J Clin Microbiol 2011 49:2502-2508

20. Gagneux S, Burgos MV, DeRiemer K, Encisco A, Muñoz S, Hopewell PC, et al: Impact of bacterial genetics on the transmission of isoniazid-resistant Mycobacterium tuberculosis. PLoS Pathog 2006, 2:e61.

21. Hazbón $M H$, Brimacombe $M$, Bobadilla Del Valle $M$, et al: Population genetics study of isoniazid resistance mutations and evolution of multidrug-resistant Mycobacterium tuberculosis. Antimicrob Agents Chemother 2006, 50:2640-2649.

22. Abe C, Kobayashi I, Mitarai S, et al: Biological and molecular characteristics of Mycobacterium tuberculosis clinical isolates with low-level resistance to isoniazid in Japan. J Clin Microbiol 2008, 46:2263-2268.

23. Guo H, Seet $Q$, Denkin S, Parsons L, Zhang Y: Molecular characterization of isoniazid-resistant clinical isolates of Mycobacterium tuberculosis from the USA. J Med Microbiol 2006, 55:1527-1531.

24. Hu Y, Hoffner S, Jiang W, Wang W, Xu B: Extensive transmission of isoniazid resistant $M$. tuberculosis and its association with increased multidrug-resistant TB in two rural counties of eastern China: a molecular epidemiological study. BMC Infect Dis 2010, 10:43.

25. Goto M, Oka S, Tachikawa N, et al: KatG sequence deletion is not the major cause of isoniazid resistance in Japanese and Yemeni Mycobacterium tuberculosis isolates. Mol Cell Probes 1995, 9:433-439.

26. Bergval IL, Schuitema ARJ, Klatser PR, Anthony RM: Resistant mutants of Mycobacterium tuberculosis selected in vitro do not reflect the in vivo mechanism of isoniazid resistance. J Antimicrob Chemother 2009 64:515-523.

27. Cardoso RF, Cooksey RC, Morlock GP, et al: Screening and characterization of mutations in isoniazid-resistant Mycobacterium tuberculosis isolates obtained in Brazil. Antimicrob Agents Chemother 2004, 48:3373-3381. 
28. Minh NN, Van Bac N, Son NT, Lien VT, Ha CH, Cuong NH, Mai CT, Le TH: Molecular characteristics of rifampin- and isoniazid-resistant Mycobacterium tuberculosis strains isolated in Vietnam. J Clin Microbiol 2012, 50:598-601.

29. Jugheli L, Bzekalava N, De Rijk P, Fissette K, Portaels F, Rigouts L: High level of cross-resistance between kanamycin, amikacin, and capreomycin among Mycobacterium tuberculosis isolates from Georgia and a close relation with mutations in the rrs gene. Antimicrob Agents Chemother 2009, 53:5064-5068.

30. Surcouf C, Heng S, Pierre-Audigier C, et al: Molecular detection of fluoroquinolone-resistance in multi-drug resistant tuberculosis in Cambodia suggests low association with XDR phenotypes. BMC Infect Dis 2011, 11:255.

31. Murase $\mathrm{Y}$, Maeda S, Yamada $\mathrm{H}$, et al: Clonal expansion of multidrugresistant and extensively drug-resistant tuberculosis, Japan. Emerg Infect Dis 2010, 16:94854.

32. Khue PM, Phuc TQ, Hung NV, Jarlier V, Robert J: Drug resistance and HIV co-infection among pulmonary tuberculosis patients in Haiphong City, Vietnam. Int J Tuberc Lung Dis 2008, 12:763-768.

doi:10.1186/1471-2334-13-275

Cite this article as: lem et al:: Resistance of Mycobacterium tuberculosis to antibiotics in Lao PDR: first multicentric study conducted in 3 hospitals. BMC Infectious Diseases 2013 13:275.

\section{Submit your next manuscript to BioMed Central and take full advantage of:}

- Convenient online submission

- Thorough peer review

- No space constraints or color figure charges

- Immediate publication on acceptance

- Inclusion in PubMed, CAS, Scopus and Google Scholar

- Research which is freely available for redistribution 